\title{
Theoretische Fundierung
}

\subsection{Wirtschaftswissenschaftliche Ansätze}

\subsubsection{Nachhaltigkeit}

Da eine einheitliche Definition der Nachhaltigkeit nach Literaturrecherche nicht vorgelegt werden kann, soll zum Verständnis des Konzepts die historische Entwicklung und deren Bedeutung bis zum heutigen Stand kurz skizziert werden. Das dieser Arbeit zugrunde gelegte Verständnis von Nachhaltigkeit orientiert sich am 3-Säulen-Modell (Gogoll \& Wenke, 2017, S. 136), welches anschließend erläutert wird. Danach wird abschließend die Nachhaltigkeitsstrategie der Bundesregierung in den Blick genommen, wobei sich auf die für die Arbeit relevanten Nachhaltigkeitsziele und -indikatoren beschränkt wird.

Der Ursprung des Begriffs der Nachhaltigkeit geht zurück auf Carlowitz, der im Rahmen der Forstwirtschaft formulierte, dass das Fällen von Bäumen nur dann ,nachhaltend [...] und beständig“ (Carlowitz, 1713, 105) sei, solange eine natürliche Regeneration gegeben sei.

Mit dem Brundtland-Report „Our Common Future“ (United Nations Commission on Environment and Development - UNCED, 1987) wurde ein Konzept zur nachhaltigen Entwicklung erarbeitet, in dem unter anderem das Handlungsprinzip der intergenerationalen Gerechtigkeit hervorgehoben wurde. Nach diesem entsteht die Verpflichtung, dass beim Verbrauch von Ressourcen zur gegenwärtigen Bedürfniserfüllung gewährleistet sein muss, dass nachfolgende Generationen ihre Bedürfnisse ebenfalls uneingeschränkt befriedigen können (UNCED, 1987). Im Jahr 1992 verabschiedete die erste weltweite Konferenz der Vereinten Nationen für Umwelt und Entwicklung mit der Agenda 21 (1992) einen Abschlussbericht, der neben der ökologischen auch die soziale und wirtschaftliche Komponente

S. M. Schrader, Erwerbsarbeit von Müttern und frühkindliche Fremdbetreuung, BestMasters, https://doi.org/10.1007/978-3-658-36287-4_2 
betonte und den Brundtland-Report weiterführte. Der Sachverständigenrat für Umweltfragen legte im Jahr 2002 ein Konzept vor, das vorwiegend für eine nachhaltige Entwicklung herangezogen wird und sich in der Literatur durchgesetzt hat. Das 3-Säulen-Modell (SRU 2002, 67 ff.), welches auch in den theoretischen Rahmen dieser Arbeit aufgenommen wird, soll nun erläutert werden. Um das Befriedigen von Bedürfnissen zu ermöglichen, müssen knappe Ressourcen verteilt werden. Diese Verteilung kann nur dann nachhaltig erfolgen, wenn sie gerecht vonstattengeht, sodass die Gerechtigkeit die moralische Grundlage der Ressourcenverteilung ist. Vom Aspekt der Verteilungsgerechtigkeit ausgehend werden dann die sozialen, ökonomischen und ökologischen Ressourcen entsprechend der Bedürfnisse verteilt. Diese entsprechen den drei Säulen des Konzepts und stehen gleichrangig nebeneinander (Sachverständigenrat für Umweltfragen - SRU, 2002, 67 ff.). Eine nachhaltige Entwicklung ist nur unter der Voraussetzung gegeben, dass alle drei Themenfelder bei Verteilungsentscheidungen berücksichtigt werden. Die folgende Abbildung zeigt das 3-Säulen-Modell der Nachhaltigkeit (Abbildung 2.1).

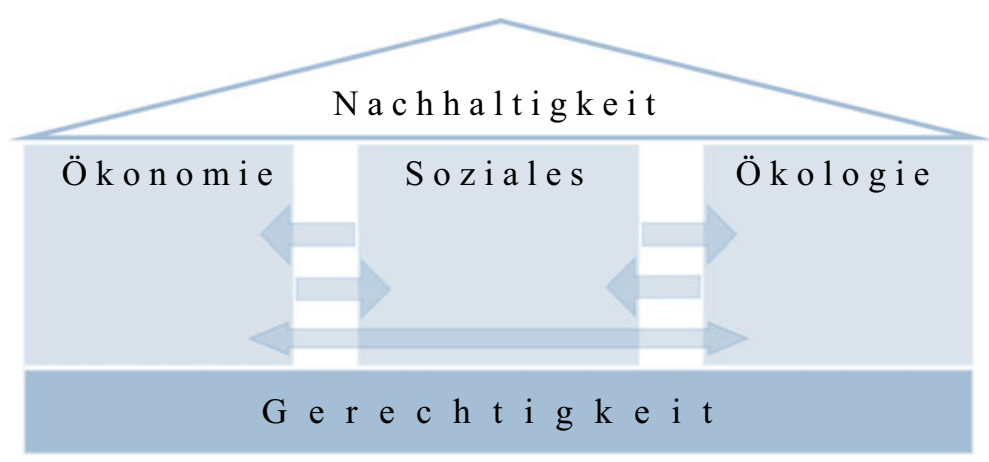

Abb. 2.1 3-Säulen-Modell der Nachhaltigkeit. (Quelle: eigene Darstellung in Anlehnung an Gogoll \& Wenke, 2017, S. 136)

Das Treffen von Verteilungs-Entscheidungen unter Berücksichtigung aller drei Bereiche verdeutlicht, dass Wechselwirkungen untereinander bestehen und sich Zielkonflikte entwickeln können, die austariert werden müssen; dies wird in der Abbildung durch die Pfeile dargestellt. Dazu sollen die Entscheidungen vom Prinzip der Verantwortlichkeit geleitet werden und im Ergebnis dauerhaft sein, da nur so eine nachhaltige Entwicklung möglich ist. 
Auf die Aspekte der Verantwortung und Gerechtigkeit, mit denen auch ein moralischer Bewertungsanspruch einhergeht (Gogoll et al., 2017, 136), wird an dieser Stelle nicht genauer eingegangen, da sie im Rahmen der Vorstellung der Begriffsdefinitionen unter 2.3 am Ende des Theorieteils gesondert behandelt werden. Es lässt sich festhalten, dass sich der Nachhaltigkeitsbegriff von einer rein ökologischen Ausrichtung hin zu einer Trias weiterentwickelt hat, die auch ökonomische und soziale Belange umfasst. In Anlehnung an die 17 im Jahr 2016 durch die United Nations (UN) verabschiedeten Sustainable Development Goals (SDG) der Agenda 2030 (United Nations Agenda 2030, 2016) hat auch die Bundesregierung im Jahr 2016 die deutsche Nachhaltigkeitsstrategie (Deutsche Nachhaltigkeitsstrategie, 2016) angepasst und Nachhaltigkeitsziele auf nationaler Ebene formuliert. Aus der aktualisierten deutschen Nachhaltigkeitsstrategie (Deutsche Nachhaltigkeitsstrategie, 2018, 53 ff.) des letzten Jahres wurden vier Nachhaltigkeitsindikatoren und Ziele ausgewählt. Sie werden in der folgenden Abbildung dargestellt und benannt und lassen sich inhaltlich den Themenfeldern des 3-Säulen-Modells zuordnen: Die Vereinbarkeit von Familie und Beruf durch den Anstieg der Ganztagsbetreuung und die Förderung der Gleichstellung betreffen das Soziale, die Schonung von Ressourcen wird der Ökologie zugeordnet und die umwelt- und sozialverträgliche Steigerung der wirtschaftlichen Leistungsfähigkeit betrifft die Ökonomie (Abbildung 2.2).

Es wird anhand der aufgeführten Indikatorenbereiche und Ziele ersichtlich, dass sich die drei Säulen der Nachhaltigkeit auch in dem Thema dieser Arbeit widerspiegeln und die Bedeutung der Wechselwirkungen und Zielkonflikte einen Untersuchungsgegenstand darstellen. Dazu soll sich, wie in der Einleitung schon erwähnt, auf die soziale Dimension konzentriert werden, wobei die Argumentation auch die ökonomische und ökologische Säule streift. Im nun folgenden Abschnitt wird die Gleichgewichtstheorie behandelt und der wirtschaftswissenschaftliche Teil des Analyserahmens damit abgeschlossen.

\subsubsection{Marktgleichgewicht}

Ein Gleichgewicht auf einem Markt entsteht immer dann, wenn die Nachfragemenge gleich der Angebotsmenge ist. Der Preis, der sich in diesem Marktgleichgewicht einstellt, ist der Gleichgewichtspreis.

Die folgende Abbildung zeigt ein Koordinatensystem und illustriert anhand der Grafik, wie sich Angebots- und Nachfragekurve schneiden. Auf der Abszisse wurde die Menge abgetragen, auf der Ordinate der Preis. Im Schnittpunkt der beiden Kurven lassen sich Gleichgewichtsmenge und Gleichgewichtspreis ablesen, 


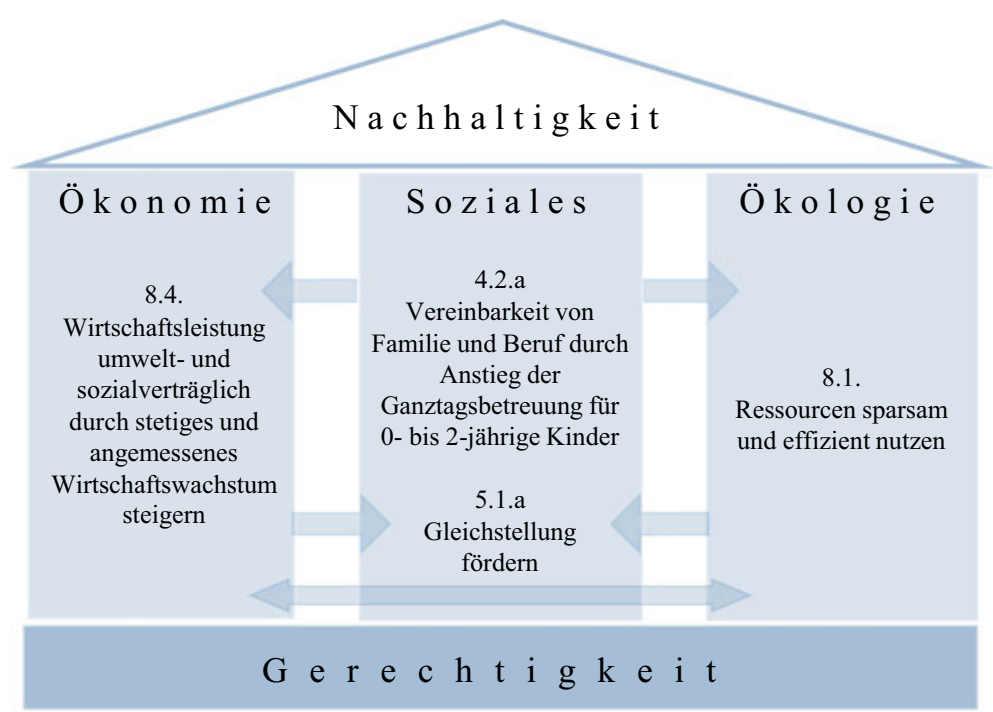

Abb. 2.2 Deutsche Nachhaltigkeitsziele im 3-Säulen-Modell der Nachhaltigkeit. (Quelle: eigene Darstellung in Anlehnung an Gogoll \& Wenke, 2017, S. 136)

dieser ist gleich dem Marktpreis. Der Markt befindet sich in einem Gleichgewicht (Abbildung 2.3).

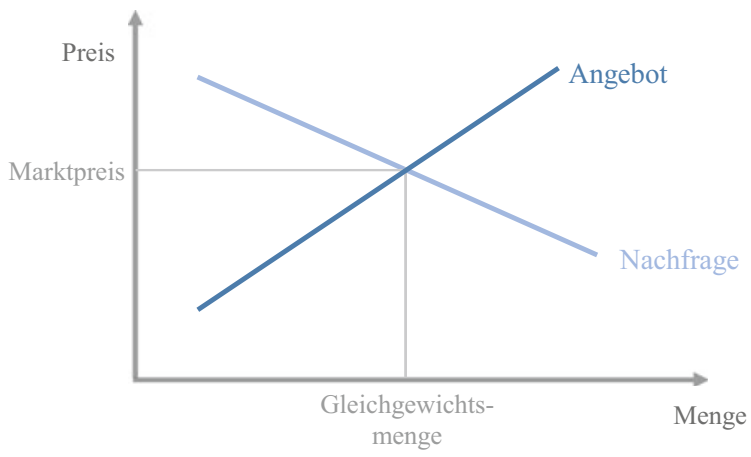

Abb. 2.3 Das Marktgleichgewicht. (eigene Darstellung) 
Das Marktgleichgewicht beschreibt im Rahmen der Theorie der Haushalte und der Unternehmen das Ergebnis effizienter individueller Tauschhandlungen. Ricardo (1817) begründete den Gedanken des Marktgleichgewichts aus der Annahme von Adam Smith (1776), der zunächst die Preisgestaltung von der Nachfrage in Abhängigkeit sah, und formulierte das erste Modell (Muhs, 1955, 47-73). Beide waren Vertreter der Nationalökonomie.

Tauschprozesse auf Märkten werden in den Volkswissenschaften untersucht und fallen unter das Teilgebiet der Mikroökonomik. Gemäß dem ökonomischen Menschenbild wird von weiteren Grundannahmen und Prinzipien ausgegangen, mit denen das individuelle Verhalten der Wirtschaftssubjekte erklärt werden kann und die Vorhersagen menschlicher Entscheidungen ermöglichen. Diese werden im Folgenden benannt (vgl. hierzu Roth, 2016). Ausgehend vom methodologischen Individualismus entscheiden die Konsumenten rational und eigennutzmaximierend, ob sie einen Tauschhandel eingehen wollen. Das individuelle Gewinnstreben orientiert sich damit an der Abwägung von Nutzen und Opportunitätskosten, die durch den Nutzen einer entgangenen Alternative entstehen. Den unbegrenzten Bedürfnissen der Marktteilnehmer stehen jedoch nur begrenzte Ressourcen zur Verfügung, sodass immer von Knappheit ausgegangen wird. Die knappen Güter müssen so effizient über Märkte auf die Bedürfnisse der Menschen verteilt werden. Dazu muss vollkommene Konkurrenz gegeben sein und vollständige Informationen vorliegen. Die Marktpreise haben in diesem Zusammenhang eine Signal- und Steuerungsfunktion, da sie die Anbieter darüber informieren, welchen Preis die Nachfrager bereit sind für die angebotenen Güter und Dienstleistungen zu bezahlen. Dies trifft auf Gütermärkte ebenso wie auf Faktormärkte zu, an denen Arbeitnehmer ihre Arbeitskraft anbieten. Die Zahlungsbereitschaft wird wiederum durch die individuellen Präferenzen der Marktteilnehmer bestimmt, die ihren Nutzen maximieren wollen. Die individuellen Präferenzen versucht jeder einzelne Marktteilnehmer mit unterschiedlichen Güterbündeln zu befriedigen, die in der Summe seinem Budget entsprechen.

Der Nutzen jeden einzelnen Gutes nimmt mit zunehmender Menge jedoch ab, wie es Gossen (1854, 4 f.) im Gesetz des abnehmenden Grenznutzens beschrieben hat. Ein Mehr an einem Gut bedeutet folglich nicht generell ein Mehr an Nutzen. Dieser kann nach Überschreiten des Sättigungspunktes auch entfallen oder negativ werden. Um alle Bedürfnisse befriedigen und nutzenstiftende Produkte am Markt anbieten zu können, hat sich Arbeitsteilung und Spezialisierung als komparativer Vorteil (Roth, 2016, 14 ff., 16 ff.) in Gesellschaften erwiesen. Durch diesen, und damit durch eine effiziente Allokation von Ressourcen, konnte die Gesamtwohlfahrt von Volkswirtschaften gesteigert werden. Wohlfahrtssteigernde, pareto-effiziente (Roth, 2016, 23 ff.) Zustände können dann erreicht 
werden, wenn durch eine Tauschhandlung eine Besserstellung erreicht werden kann, ohne eine andere Person damit schlechter zu stellen. Da die Marktteilnehmer in Freiwilligkeit handeln und keinen Tausch zu ihren Ungunsten eingehen würden, kann geschlossen werden, dass ein Marktgleichgewicht zu allgemeinem Wohlfahrtszuwachs führt.

Da die Betrachtung von Wohlfahrtseffekten in Bezug auf die Erwerbstätigkeit von Müttern und die frühkindliche Fremdbetreuung Ziel dieser Arbeit ist, wird das Modell des Marktgleichgewichts zur Erklärung des Verhaltens von Müttern herangezogen. Dabei soll sich nicht nur auf den Arbeitsmarktkontext bezogen werden, sondern auch auf Tauschhandlungen zwischen Mutter und Kind. Dafür soll das Modell des Marktgleichgewichts mit dem psychologischen Ansatz des „Giving vs. Giving In“ verknüpft werden und diesen erweitern. Er wird im nun folgenden Abschnitt vorgestellt und eröffnet den psychologischen Teil des Analyserahmens.

\subsection{Psychologische Ansätze}

\subsubsection{Giving vs. Giving In}

Zwischen Geben und Geben besteht auf den ersten Blick kein Unterschied, auf den zweiten Blick hingegen schon. Das belegten Cain, Dana und Newman (2014) in einer Studie zu prosozialem Verhalten. Sie stellten fest, dass ca. $50 \%$ offensichtlich altruistischen Verhaltens eigentlich widerwillig geschah.

Die Autoren untersuchten Daten anhand einer eigenen Laborstudie und werteten zusätzlich Feldstudien zu altruistischem Verhalten aus. Es zeigte sich, dass im Gegensatz zur evolutionsbiologischen Definition prosozialen Verhaltens, durch Reduktion eigener Ressourcen die eines anderen in der Konsequenz zu erhöhen (Fetchenhauer, 2011, 436), unterschiedliche Motive zugrunde liegen. Mit „Giving“ geht ein Pflichtgefühl einher, einem anderen bereitwillig Vorteile zu verschaffen. Während es beim ,Giving In“ dem Gebenden widerstrebt, für andere etwas zu tun und er sich dazu gezwungen fühlt. Die Frage nach dem Evolvieren von altruistischem Verhalten beantworten die wissenschaftlichen Disziplinen sehr unterschiedlich (Pradel, 2010). In der Ökonomie wird, wie schon erwähnt, vom Eigennutzmotiv einer Person ausgegangen, während in der Biologie eine Strategie, das Überleben sicherzustellen, für wahrscheinlich gehalten wird. In der Psychologie wird diskutiert, dass Gruppen von Altruisten Vorteile gegenüber Egoisten haben, weil sie sich untereinander erkennen (Frank, 1988), und 
ob das Geben letztendlich nur geeignet ist, zu einem positiven Selbstbild beizutragen. Die Autoren führen unterschiedliche Erklärungen an. So hat Reziprozität einen positiven Reputationseffekt, der anderen signalisiert, dass die Bereitschaft zu kooperativem Verhalten vorliegt. Das gilt auch dann, wenn diese gar nicht beabsichtigt ist, also eigentlich Giving In vorliegt. Ein weiteres Argument ist, dass Giving nach außen Generosität demonstriert, welches dem Selbstimage dient und extrinsisch motiviert ist. Das altruistische Geben kann auch dazu führen, dass im Gehirn das Belohnungszentrum aktiviert wird und sich dadurch das Verhalten wiederholt. Als weiteren Grund nennen Cain et al. (2014) die Orientierung an sozialen Präferenzen, um sich gemäß Giving oder Giving In zu verhalten. Die Autoren konnten zwei Strategien identifizieren, nach denen Personen verfahren, um Kontexte zu vermeiden, in denen sie sich zu prosozialem Verhalten gezwungen fühlen. Zum einen gelingt dies durch die Exit-Option, mit der vermieden werden kann, dass andere erfahren, dass man hätte helfen können. Zum anderen wird das Vermeiden durch strategische Ignoranz (Grossman, 2014) ermöglicht, in dem sich bewusst nicht über das Hilfeersuchen eines anderen informiert wird. Zudem hat sich ein Einfluss von Persönlichkeitsmerkmalen auf das Verhalten gezeigt, so dass eine Sensibilität für Gerechtigkeit die Wahrscheinlichkeit prosozialen Verhaltens erhöht. Überwiegend dominiert jedoch das Giving In, das widerstrebende prosoziale Verhalten.

Die wenigsten Menschen lassen sich allerdings dauerhaft zur Gruppe der „Giver“ (Altruisten) oder „Taker“ (Egoisten) zuteilen. Der Großteil, die „Matcher“, lassen sich in ihrem Verhalten vom Kontext beeinflussen und passen sich an. Öffentlich beobachtet oder unmittelbar angesprochen zu werden, führt bei ihnen zu helfendem Verhalten, während sie durch Verantwortungsdelegation vermeiden, sich die Konsequenzen ihres eigenen Handelns vor Augen zu führen. Die Autoren konnten ebenfalls den Einfluss sozialer Normen auf das Verhalten belegen. Studierende der Wirtschaftswissenschaften beurteilten und zeigten sich mit zunehmender Studiendauer egoistischer und entsprachen so dem eigennutzmaximierenden ökonomischen Menschenbild, welches im vorangegangenen Abschnitt bereits genauer besprochen wurde, und damit den Erwartungen ihres sozialen Umfelds.

Um die Frage nach Wohlfahrtseffekten beantworten zu können, ist eine theoretische Einordnung altruistischer Motive im Kontext der Wiederaufnahme der Erwerbsarbeit von Müttern und institutioneller Unterbringung der Kleinkinder erforderlich. Durch eine Betrachtung der Schnittstelle unterschiedlicher Perspektiven, die der Kinder, Mütter und Erzieher, lassen sich im Hinblick auf die Ergebnisse des zuvor vorgestellten Ansatzes Erklärungen für Erleben und Verhalten ableiten. Dabei sind Bereitwilligkeit und strategische Ignoranz ebenso von 
Bedeutung wie der Einfluss sozialer Normen und die direkte Ansprache. Unter Einbezug des wirtschaftswissenschaftlichen Ansatzes des Marktgleichgewichts, Nachfrage und Angebot individueller Präferenzen und Bedürfniserfüllung, soll der Ansatz des „Giving vs. Giving In“, wie bereits angedeutet, weiterentwickelt werden.

Da Hilfeverhalten durch unmittelbare Ansprache ausgelöst werden kann, stellt sich die Frage nach einer Kommunikationsform für den Fall, in dem die Sprache (noch) fehlt. Da dieser Fall regelmäßig auf Kleinkinder zutrifft, deren Spracherwerb entwicklungsbedingt noch nicht dem älterer Kinder oder Erwachsener gleicht, wird im kommenden Abschnitt erläutert, wie Kommunikation zwischen Mutter und Kind trotzdem gelingen kann und die Begriffe Feinfühligkeit und intuitive elterliche Kompetenz eingeführt.

\subsubsection{Feinfühligkeit und Intuitive elterliche Kompetenzen}

Die Art und Weise, wie sensibel die Bindungsperson auf die Signale eines Kindes eingeht, beschreibt das bindungstheoretische Konzept der Feinfühligkeit (Ainsworth, 1974). Dieses Konzept basierte auf den Befunden Bowlbys zu dissozialem Verhalten nach Fremdunterbringung und Verlust der Bindungsperson, über die er bereits im Jahr 1952 die WHO unterrichtet hatte. Papoušek und Papoušek (2002, 183-206) entwickelten das Konzept der Feinfühligkeit weiter und untersuchten vertieft die intuitiven Kompetenzen der Eltern. Der Grad der Feinfühligkeit intuitiver elterlicher Kompetenzen bestimmt die Qualität der späteren Bindung.

Ainsworth (1974) beschreibt die Feinfühligkeit als frühe Form der vorsprachlichen Kommunikation und Kooperation zwischen der frühen Bindungsperson, meist der Mutter, und dem Kind. Dazu muss die Bindungsperson in der Lage sein, die Signale des Kindes zum einen wahrzunehmen und zum anderen auch entsprechend des mitgeteilten Bedürfnisses des Kindes zu interpretieren. Unmittelbar danach muss eine Antwort der Bindungsperson auf dieses kindliche Signal erfolgen, die dem vermittelten Bedürfnis angemessen ist. Die Autorin betont die Wichtigkeit der dazu erforderlichen emotionalen Verfügbarkeit der Eltern und deren Akzeptanz der kindlichen Bedürfnisse nach Nähe und Sicherheit. Zur Beurteilung der Ausprägung der elterlichen, vorsprachlichen Kompetenzen entwickelte Ainsworth (1974) eine neunstufige Skala, die das Spektrum zwischen Feinfühligkeit und Unfeinfühligkeit abdeckt sowie auch den „Fremde-Situations-Test“ (Grossmann \& Grossmann, 2012, 136 ff.), anhand dessen sich unterschiedliche Bindungsmuster zeigen und eine Beurteilung der Bindungsqualität zwischen Mutter und Kind erlauben. Es werden vier Bindungstypen unterschieden. Die sichere 
Bindung wird als die entwicklungspsychologisch günstigste beurteilt (Grossmann et al., 2012, 140 ff.), da sie anzeigt, dass das Kind seine Mutter als verlässliche Basis ansieht.

Wie zu Beginn erwähnt, wurde das feinfühlige Verhalten der Eltern in Bezug auf ihre intuitiven Kompetenzen eingehend durch Papoušek et al. (2002, 183-206) untersucht. Sie konnten anhand der Analyse von Videosequenzen belegen, dass das Antwortverhalten der Eltern auf Signale des Kindes im Millisekundenbereich erfolgte, hoch motiviert und für diese nicht ermüdend war. Die Eltern setzten zur Kommunikation Mimik, Gestik und auch sprachliche Mittel ein und ließen sich dabei von den wahrgenommenen kindlichen Signalen unbewusst leiten. Ihr Verhalten war sowohl am Alter des Kindes als auch an der Situation orientiert. Sie dosierten das Wechselspiel in angemessener Weise, anregend oder beruhigend, am kindlichen Erregungsniveau und unterstützten es bei seiner Emotionsregulation (Grossmann et al., 2012, 117 f.) und dem Abbau von Stress. Papoušek et al. (2002, 183-206) konnten beobachten, dass das Verhalten der Eltern für den Säugling vorhersehbar war und er diesbezüglich eine motivationale Erwartungshaltung ausbilden konnte, ebenso wie auch die Eltern. Die Erfüllung der Erwartungshaltung sorgt dafür, dass sich Kompetenz- und Selbstwirksamkeitsgefühl des Säuglings positiv entwickeln.

Die Forscher konnten ebenfalls die Dynamik des vorsprachlichen Kommunikationsgefüges unter Beteiligung neurobiologischer Aktivitäten erklären. Die Aktivierung von Spiegelneuronen ermöglicht unwillkürliches Nachahmen und führt selbst dann zu einer neuronalen Verarbeitung, wenn nur beobachtet wird (Berk, 2011, 178). Zudem wird durch die Interaktionen das Bindungshormon Oxytocin ausgeschüttet sowie das dopaminerge Motivations- und Belohnungszentrum aktiviert. Die auf diese Weise ausgelösten Rückkopplungsprozesse lösen wiederum synaptisches Wachstum aus und formen kognitive Spuren (Grossmann et al., 2012, 63 ff.). Die Autoren gehen von einem universellen und implizit vorliegenden elterlichen Wissen aus, sich bei der Erarbeitung des Kommunikationssystems mit ihrem Kind auf dessen Signale einzulassen und an ihnen zu orientieren. Auf diese Weise festigt sich die Beziehung zwischen Eltern und Kind, die eine gesunde frühkindliche Entwicklung fördert und im weiteren Verlauf als Ressource fungiert.

In Bezug auf den Wechsel in die Fremdbetreuung ist ein Vergleich der Kompetenzen hinsichtlich der Feinfühligkeit von Müttern und Erziehern vorzunehmen und auf die kindlichen Bedürfnisse und Erwartungen hin zu untersuchen, da die Erzieher die Kommunikation mit dem Kind während der Abwesenheit der Mutter übernehmen. Dass dabei die beiderseitige Motivation eine wichtige Rolle spielt, wurde bereits dargelegt. Aus diesem Grund wird im kommenden Abschnitt der 
Unterschied zwischen intrinsischer und extrinsischer Motivation erläutert und in den Analyserahmen aufgenommen.

\subsubsection{Intrinsische vs. Extrinsische Motivation}

„Die Motivation einer Person, ein bestimmtes Ziel zu verfolgen, hängt von situativen Anreizen, persönlichen Präferenzen und deren Wechselwirkung ab (Heckhausen \& Heckhausen, 2011, 7). Ein durch Wollen initiiertes Handeln geschieht entweder aufgrund des Wunsches ein Ziel zu erreichen, dann ist es ein Mittel zum Zweck und damit extrinsisch motiviert, oder aufgrund der Freude an einer Tätigkeit selbst, dann ist diese selbst der Zweck und intrinsisch motiviert (Fetchenhauer, 2011, 62; Rudolph, 2013, 153).

Die Anfänge der Motivationspsychologie gehen zurück auf Epikur (341-270 v. Chr.), dessen hedonistisches Prinzip, Maximieren der Freude bei Minimieren eigenen Leids, es war, die Frage nach den Beweggründen menschlicher Handlungen zu beantworten (Rudolph, 2013, 14). Seitdem wurden zahlreiche Theorien hervorgebracht. Die für die Untersuchung der Fragestellung dieser Arbeit entscheidenden Aspekte sollen von diesen Theorien jedoch isoliert betrachtet werden, da sie für das Verständnis nicht erforderlich sind. Im Folgenden werden nun zwei weitere Teilaspekte hinsichtlich der Motivation erläutert, die im Zusammenhang mit der Fragestellung von Bedeutung sind.

Zum einen können Motive hinsichtlich der sozialen Komponente unterschieden werden. Hierzu wird sich an der Einteilung von Fetchenhauer (2011, 58-62) orientiert und die Motive in Nichtsoziale und Soziale unterteilt. Die nichtsozialen Motive entsprechen den physiologischen Grundbedürfnissen von Menschen und sichern deren Existenz. Dazu gehört der Wunsch nach Nahrung sowie körperlicher Unversehrtheit. Das Bedürfnis nach Kontrolle der Umwelt und Unabhängigkeit ist besonders stark ausgeprägt und erklärt den Drang nach Exploration und Kompetenzerwerb, der sich bereits sehr früh bei Kleinkindern zeigt. Es handelt sich dabei um Wachstumsmotive (Schnotz, 2006, 89 f.), die eng in Bezug zum Selbstwirksamkeitserleben (Bandura, 1986) stehen. Die Grundvoraussetzung der späteren Selbstwirksamkeit sehen Heckhausen et al. $(2011,9)$ im Kompetenzerwerb während des Säuglings- und frühen Kindesalters. Die Selbstwirksamkeit spielt auch im Hinblick auf die sozialen Motive eine wichtige Rolle, da das Bedürfnis nach Zugehörigkeit zu einer sozialen Gruppe eines der stärksten und grundlegendsten menschlichen Motive (Fetchenhauer, 2011, 60) ist. Das Gefühl dazuzugehören, löst positive Emotionen aus. So führt die Anerkennung der Gruppenmitglieder zu einem Erleben von Freude und Stolz, während das 
Empfinden von Angst und Verzweiflung einen Ausschluss aus einer Gruppe oder eine Nichtzugehörigkeit signalisiert. Die Empfindungen sind dabei auch durch soziale Normen determiniert, was Fetchenhauer (2011, 60 f.) beispielhaft an der Kulturabhängigkeit des Schamgefühls besonders betont. Weitere in Zusammenhang mit der Arbeit stehende, bedeutende soziale Motive sind neben sozialem Status und Selbstwert (Fetchenhauer, 2011, 420 ff., 303 ff.; Rudolph, 2013, 140) auch das Fürsorgemotiv von Frauen (Fetchenhauer, 2011, 191).

Zum anderen unterscheidet Fetchenhauer (2011, 63-65) Motive auch danach, ob sie Personen bewusst sind oder unbewusst vorliegen. Während bei expliziten, das heißt bewussten, Motiven eine genaue Vorstellung dieser benannt werden kann, sind implizite, also unbewusste Motive, der Introspektion nicht zugänglich. Die Erfassung anhand einer Selbstbefragung ist daher nicht möglich und gelingt nur indirekt über die Operationalisierung von Bildern und deren Interpretation. Explizit motivierte Handlungen können durch lohnende externe Anreize beeinflusst werden und stellen die extrinsische Motivation dar. Hingegen liegt bei implizit motiviertem Tun der Anreiz in der Erreichung eines selbst gewählten Zielzustands und entspricht der intrinsischen Motivation. Beide Motivformen können allein oder auch in Kombination miteinander vorkommen, wobei eine völlige Übereinstimmung selten der Fall ist (Fetchenhauer, 2011, 64).

Menschen sind motiviert, wie schon zu Beginn erwähnt, positive Emotionen anzustreben sowie negative möglichst zu vermeiden (Fetchenhauer, 2011, 53). Da Gefühle sich der bewussten Kontrolle entziehen, informieren sie darüber, ob eine Annäherung oder eine Entfernung von einem Zielzustand vorliegt. Während positive Emotionen sich nur kurz einstellen, ist die Persistenz negativer Emotionen anhaltender und das Empfindungsspektrum größer (Fetchenhauer, 2011, 70); durch diese Affekte informiert sich eine Person über die Stärke eines Motivs quasi selbst. Da Affekte nur subjektiv empfunden, interpretiert und bewertet werden können, ist eine objektive Beurteilung nicht möglich. Dem Handlungsergebnis werden Ursachen zugeschrieben, die entweder extern in den Umständen liegen oder intern auf eine Person attribuiert werden (Heckhausen et al., 2011, 7; Rudolph, 2013, 134). Eine positive Selbstwirksamkeitserwartung kann dann entstehen, wenn positive Handlungsfolgen der eigenen Kompetenz zugeschrieben und antizipiert werden (Schnotz, 2006, 94), wodurch sich die Anstrengungsbereitschaft erhöht.

Zusammenfassend lässt sich festhalten, dass Motive durch äußere oder innere Anreize entstehen, die bewussten Motive kaum mit den unbewussten übereinstimmen und dass soziale Normen einen erheblichen Einfluss auf die Motivation von Personen haben. Um die Frage nach Wohlfahrtseffekten beantworten zu können, sollen die erläuterten Aspekte der Motivation herangezogen werden, um 
die Motive von Kindern, Müttern, und Erziehern zu untersuchen. Einmal hinsichtlich des Betreuungswechsels, zum anderen hinsichtlich des Wiedereinstiegs in den Beruf und der Erzieherrolle. Dabei ist eine Betrachtung des Kontrollbedürfnisses wichtig, die der Subjektivität der Empfindungen und das Vorliegen unterschiedlicher Anreize.

Nicht nur Anreize allein bedingen die Anstrengungsbereitschaft von Personen, sondern vielmehr auch die Erwartung von Belohnung und Strafe. Daher wird dieser Aspekt im nächsten Abschnitt vertiefend betrachtet und die Sozialkognitive Lerntheorie vorgestellt, die das Lernen durch Vorbilder erklärt und den psychologischen Teil des Analyserahmens abschließt.

\subsubsection{Lernen am Modell}

Durch die Beobachtung anderer wird Verhalten gelernt. Die Wahrscheinlichkeit der Nachahmung ist abhängig davon, ob die beobachtete Person für ihr Verhalten im Anschluss belohnt oder bestraft wurde.

Die Sozial-kognitive Lerntheorie (Bandura, 1979, 1965), auch Lernen am Modell genannt, hat die behavioristischen Ansätze um die kognitive Komponente entscheidend erweitert (Edelmann \& Wittmann, 2012, 165-168). Bandura (1979, 1965) konnte die Bedeutung kognitiver Lernvorgänge auf Verhalten durch Beobachtung anhand eines Experiments nachweisen, dass er gemeinsam mit Walters im Jahr 1965 durchführte. Innerhalb der „Bobo doll study“, die durch das Rocky-Experiment bekannt wurde, führte er drei Kindergruppen einen Film vor, in dem eine erwachsene Person (Rocky) eine Plastikpuppe (Bobo) aggressiv behandelte und beschimpfte. Die Untersuchungsbedingungen unterschieden sich dadurch, dass der Erwachsene am Ende der Filmsequenz einmal eine Belohnung erhielt, einmal bestraft wurde und einmal keine Konsequenz auf das aggressive Verhalten folgte. Danach wurden die Kinder einzeln zum Spielen in einen Raum geführt, in dem sie auch die Plastikpuppe vorfanden, die sie zuvor im Film gesehen hatten. Die Kinder, die die Belohnung des Erwachsenen beobachtet hatten und das Erfolgen keiner Konsequenz, zeigten der Puppe gegenüber signifikant häufiger das aggressive Verhalten, dass sie zuvor im Film gesehen hatten, als die Kinder, die eine Bestrafung der erwachsenen Person beobachtet hatten. Auf Befragung hin, was die Kinder im Film gesehen hätten, konnten sich die Kinder aller Gruppen gleichermaßen an das aggressive Verhalten des Erwachsenen erinnern und es beschreiben. Im Anschluss daran wurde den Kindern aller Gruppen 
eine Belohnung versprochen, wenn sie sich gegenüber der Puppe aggressiv verhalten würden. Dies führte dazu, dass die Kinder aller Versuchsbedingungen der Puppe gegenüber verstärkt aggressives Verhalten zeigten.

Hieraus konnte Bandura $(1979,1965)$ schließen, dass allein durch Beobachtung kognitive Lernprozesse bei den Kindern stattgefunden haben, in denen sie sich das Verhalten, unabhängig von den Konsequenzen der einzelnen Versuchsbedingungen, angeeignet haben. Das Nachahmen des aggressiven Verhaltens war davon abhängig, welche Konsequenz die Kinder für sich antizipierten und entschieden entsprechend ihrer Erwartung, ob sie sich gegenüber der Puppe ebenfalls aggressiv verhalten wollten oder nicht. Entsprechend seiner Ergebnisse unterteilte Bandura $(1979,1965)$ den Vorgang des sozial-kognitiven Lernens in zwei Phasen, die Akquisition (Aneignung) und die Performanz (Ausführung).

Die Phase der Akquisition unterteilte er weiter und betonte zum einen die Notwendigkeit der Aufmerksamkeitsprozesse durch eine differenzierte Wahrnehmung, die Förderung der Aufmerksamkeit durch prestigeträchtige und kompetente Modellpersonen sowie ein gutes Beziehungsverhältnis und die emotionale Erregung des Beobachtenden. Zum anderen betonte er die aktiven Gedächtnisprozesse des Lernenden durch Verarbeitung und Kodierung der Reize in kognitive Schemata vor deren verbaler und bildlicher Speicherung, wodurch sich das beobachtete Verhalten auch zeitlich verzögert zeigen konnte.

Die Phase der Performanz unterteilte Bandura $(1979,1965)$ in motorische Reproduktionsprozesse, die über die kognitiven Repräsentationen des Beobachters abgerufen werden, und in Motivationsprozesse. Diese sind abhängig von den antizipierten Konsequenzen durch positive oder negative Verstärkung, das heißt Belohnung oder Bestrafung. Motivationale Konflikte können durch Effizienzerwartungen (Fetchenhauer, 2011, 84 f.) des Beobachters und die Differenz von Fremd- und Selbstbewertung entstehen und dessen Verhalten entsprechend seines Selbstkonzepts (Bandura, 1993) beeinflussen.

In Bezug zur Fragestellung ist eine Betrachtung der Auswirkungen aufgrund des stattfindenden Wechsels des Betreuungssettings aus kindlicher Perspektive zu untersuchen sowie die Frage nach möglichen Formen des Beobachtungslernens durch die Mütter. Dazu sollen Belohnung und Bestrafung ebenso in den Blick genommen werden wie Effizienzerwartungen, Selbstkonzept und Kognitionen.

Zur Ergänzung des hiermit abgeschlossenen Analyserahmens, bestehend aus wirtschaftswissenschaftlichen und psychologischen Theorien, sollen im kommenden Abschnitt zunächst zwei Begriffsdefinitionen vorgenommen werden. Hiernach erfolgt die aus Abschnitt 2.1.1 hinsichtlich der Nachhaltigkeit noch ausstehende gesonderte Betrachtung von Verantwortung und Gerechtigkeit, die durch die moralische Urteilsbildung im Anschluss ergänzt wird. 


\subsection{Begriffsdefinitionen}

\subsubsection{Frühkindliche Fremdbetreuung}

Im Kontext dieser Arbeit soll unter frühkindlicher Fremdbetreuung die institutionelle Unterbringung von Kleinkindern im Alter bis zu drei Jahren (U3) verstanden werden, die von erwerbsmäßig beschäftigten Fachkräften geleistet wird. Sie wird auf Bundesebene im Sozialgesetzbuch Achtes Buch (SGB VIII), zweites Kapitel, dritter Abschnitt, vom Jahr 1990 geregelt, dem Kinder- und Jugendhilfegesetz (KJHG). Die Begriffsbildung ist in der Literatur uneindeutig und es werden verschiedene Ausdrucksformen für die außerfamiliäre Kinderbetreuung verwendet. So finden Begriffe wie Kindertagesbetreuung, Kindergarten, Tagesbetreuung oder Ganztagsbetreuung synonym Verwendung. Kindertagespflege und Tagespflege bezieht sich hingegen auf ein privat organisiertes Betreuungsarrangement mit Tagesmüttern, von dem sich in dieser Arbeit ebenso abgegrenzt wird, wie von der durch Verwandte des Kindes geleistete Betreuung. Der Begriff Kinderkrippe, Krippengruppe, oder auch Krippe, ist mit der hier verstandenen Bedeutung frühkindlicher Fremdbetreuung jedoch gleichzusetzen, da er in der Deutschen Demokratischen Republik für die Tagesbetreuung unter Dreijähriger üblich war und in den westlichen Bundesländern zunehmend verwendet wird.

Die Begriffsbestimmung der frühkindlichen Fremdbetreuung ist in Zusammenhang mit der Untersuchung der Fragestellung relevant, da die Effekte untersucht werden sollen, die aufgrund der Betreuung durch hauptberufliche Erzieher in institutionellen Einrichtungen entstehen. Im Gegensatz zu Verwandten, die die Versorgung der Kleinkinder in der Regel unentgeltlich übernehmen, beziehen Erzieher ein Gehalt für ihre Tätigkeit und unterscheiden sich dadurch auch motivational.

\subsubsection{Vollzeittätigkeit}

Für die Bestimmung eines vollerwerbstätigen Arbeitsumfangs wird die normative Grundlage des Arbeitszeitgesetzes (ArbZG) herangezogen, die in $\S 3$ eine durchschnittliche werktägliche Arbeitszeit der Arbeitnehmer von acht Stunden festlegt. Diese muss durch Ruhepausen ( $\$$ ) unterbrochen werden. Im Anschluss an die tägliche Arbeitszeit steht den Arbeitnehmern eine ununterbrochene Ruhezeit ( $\S$ 5) von mindestens elf Stunden zu (ArbZG, 1994).

Das Arbeitszeitgesetz dient unter anderem dem Zweck (§ 1) des Gesundheitsschutzes der Arbeitnehmer durch zeitliche Überlastung (ArbZG, 1994). 
Deshalb werden arbeitsfreie Zeiten sowie die generelle Sonn- und Feiertagsruhe (§ 9) vorgeschrieben, die der Erholung dienen sollen. Ausnahmen von den verbindlichen Vorgaben sind unter der Voraussetzung eines zeitlichen Ausgleichs möglich ( $\$ 7$ Abs. 2 ArbZG, 1994). Arbeitszeiten, die durch die Arbeit bei mehreren Arbeitgebern entstehen, müssen dabei zusammengerechnet werden $(\S$ 2), sodass die Höchstzahl an Arbeitsstunden nicht überschritten wird (ArbZG, 1994). Neben der Vollzeittätigkeit mit einer wöchentlichen Arbeitszeit von 40 Stunden, existiert auch ein Recht auf Teilzeitbeschäftigung. Ziel des Teilzeitund Befristungsgesetzes (TzBfG) ist es, die Teilzeitarbeit zu fördern ( $\$ 1$ ). Die Wochenarbeitszeit unterschreitet dann regelmäßig das bei Vollbeschäftigung geltende Maß an Arbeitsstunden ( $\$ 2$ TzBfG, 2000).

Die ordnungspolitische Regelung spiegelt den Fürsorgeauftrag des Staates für die Bürger wider, indem er zum gesundheitlichen Schutz der Arbeitnehmer eine Obergrenze des Erwerbsumfangs festlegt. Ein Vergleich der Arbeitsbelastung und Erholungs- und Ruhezeiten zwischen Müttern und Kinderlosen ist notwendig, um mögliche Wohlfahrtseffekte untersuchen zu können, da die Betreuung von Kleinkindern auch während ,,arbeitsfreier" Zeiten sichergestellt werden muss und Mütter diesbezüglich in der Verantwortung stehen.

\subsubsection{Verantwortung}

,Verantwortung ist die als Pflicht anerkannte Sorge um ein anderes Sein, die bei Bedrohung seiner Verletzlichkeit zur Besorgnis wird“ (Jonas, 2017, 391). Verantwortung setzt die Möglichkeit freiheitlich $\mathrm{zu}$ entscheiden und $\mathrm{zu}$ handeln voraus, sodass Handlungen und ihre Folgen der freiheitlich Handelnde selbst zu verantworten hat (Gogoll et al., 2017, 29; Noll, 2013, 52).

Der handelnde Mensch ist jedoch nicht nur zur Verantwortung gegenüber anderen verpflichtet, sondern er trägt auch sich selbst gegenüber die Verantwortung. Das Treffen moralischer Entscheidungen, die durch Anwendung ethischer Theorien nicht abgenommen werden kann, stellt eine individuelle Last des Verantwortlichen dar (Popper, 1980, 111 in Noll, 2013, 41). Grundsätzlich ist bei der Bewertung von Handlungsfolgen zu berücksichtigen, dass es sich um vielschichtige Beziehungen handelt. Noll $(2013,53)$ interpretiert den Verantwortungsbegriff danach, welche Person die Verantwortung trägt, wofür genau diese sie trägt und gegenüber wem, und dass sich die Bewertung der Handlungsfolgen an einem normativen Kriterium vollzieht. Diese Instanz kann gesetzlich vorgegeben sowie moralischer Natur sein und sich an sozialen Normen orientieren. Die Verantwortlichkeit folgt dem Grundsatz, dass dem Sollen immer auch ein Können und 
Wollen vorausgesetzt sein muss (Fenner, 2008, 175; Gogoll et al., 2017, 32) und trifft auf Einzelpersonen ebenso zu wie auf Gruppen. Dabei gilt das Subsidiaritätsprinzip und verpflichtet eingreifende Gruppen einen Beleg über den Mehrwert ihres Handelns zu erbringen (Noll, 2013, 51).

Jonas' Prinzip der Verantwortung (2017) soll für diese Arbeit herangezogen werden, da sich die elterliche Fürsorge ,permanent“ auf alles bezieht, was an einem Kind „betreubar“ ist (Jonas, 2017, 178) und die Ur-Verantwortung (Jonas, 2017, 185, 234) der Eltern darstellt. Dazu soll eine gedankliche Brücke zur Transition der frühkindlichen institutionellen Kinderbetreuung (UNICEF, 2008) geschlagen werden, durch die sich die Aufwachs- und Entwicklungsbedingungen von Kleinkindern in den hochentwickelten Ländern grundlegend geändert haben und sich weiter im Wandel befinden. Jonas $(2017,70)$ empfiehlt bei der Abschätzung möglicher Folgen einer neuen Technologie der schlechten Prognose mehr Gewicht vor der guten einzuräumen und betont den präventiven Einfluss der menschlichen Verantwortung. Daher erscheint der technologische Fortschritt in der „Produktion des Produktionsfaktors Arbeit“, die frühe institutionelle Fremdbetreuung von Kleinkindern der mütterlichen vorzuziehen, geeignet, um die Überlegungen Jonas' zu übertragen und auf die vorliegende Themenstellung anzuwenden. Durch die Übertragung der Verantwortung von der Mutter auf die Erzieher für die Dauer der täglichen Erwerbsarbeit, ist der Staat letztendlich für das Wohl der Kinder und die sich ergebenden Folgen verantwortlich. Es stellt sich aber in Zusammenhang mit dem Betreuungswechsel der Kinder nicht nur die Frage nach der Verantwortung, sondern auch die der Gerechtigkeit, da sich die Allokation von Ressourcen verändert, und insbesondere die der mütterlichen Zeit.

\subsubsection{Gerechtigkeit}

Gerechtigkeit ist ein universales ethisch-moralisches Prinzip, nachdem wechselseitige soziale Interaktionen und ihre Ergebnisse beurteilt werden (Fenner, 2008, 189; Höffe, 2004, 34).

Es wird sich auf die psychologische Perspektive der Gerechtigkeit (Gollwitzer \& Prooijen van, 2016, 61-82) konzentriert und damit am psychologischen Theorierahmen der Arbeit orientiert, weil der soziale Aspekt im Mittelpunkt der Betrachtung steht. Der Gerechtigkeitspsychologie liegen unterschiedliche Theorien zugrunde. Im Folgenden wird der Einordnung von Fetchenhauer (2011, $441 \mathrm{ff}$.) gefolgt, da sich alle für die Arbeit wesentlichen Punkte daran herleiten lassen. 
Fetchenhauer (2011, 441 ff.) nimmt eine Einordnung vor, die sich an der grundsätzlichen Unterteilung zwischen distributiver und prozeduraler Gerechtigkeit (Walzer, 2006, 51 ff.) orientiert und daraus weitere Prinzipen ableitet. Der Verteilungsgerechtigkeit liegen drei weitere Prinzipien zugrunde, nach dem knappe Ressourcen verteilt werden können. Zum einen orientiert sich das Gleichheitsprinzip an der Leistung und legt eine egale Verteilung von Gütern zugrunde, unabhängig von der Höhe der eingebrachten Leistung in die Gemeinschaft. Diese Gleichheit bezieht sich auch auf die kommutative Gerechtigkeit (Fenner, 2008, 190). Die Äquivalenz von freiwillig getauschten Gütern erfüllt die Gerechtigkeitsnorm. Dem Bedürfnisprinzip entsprechend, entscheidet sich die Zuteilung der Ressourcen zum anderen nach dem Anspruch, dem individuelle Bedürfnisse zugrunde liegen, welche nach diesem Gerechtigkeitsprinzip berücksichtigt werden müssen (Fenner, 2008, 192). Die Verteilung entsprechend des EquityPrinzips orientiert sich dagegen am Anteil der individuell eingebrachten Beiträge im Verhältnis der anderen Gemeinschaftsmitglieder (Fetchenhauer, 2011, 442). Die Verteilung einer knappen Ressource kann jeweils immer nur nach einem distributiven Prinzip vorgenommen werden. Dadurch ist ein Verteilungsergebnis nur aus Sicht eines angewandten Prinzips gerecht, während es am Maßstab eines anderen Prinzips als ungerecht bewertet werden muss, sodass eine Verteilung nach Leistung beispielsweise aus der Bedürfnisperspektive nicht gerecht sein kann.

Die prozedurale Gerechtigkeit unterscheidet zwei Prinzipien voneinander, die für das Gerechtigkeitsempfinden von Personen eine entscheidende Rolle spielen (Fetchenhauer, 2011, 443). Zum einen nennt er den Einfluss auf die Regeln der Entscheidungsfindung, zum anderen auf die Entscheidung als solche. Um die Bedürfnisse aller Betroffenen gleichberechtigt einbeziehen und gerechte Zustände begründen bzw. beurteilen zu können, empfahl Rawls (1996, 159 f.) eine offene Diskussion unter Ausschluss des Wissens eigenen zukünftigen Leistungsvermögens und gesellschaftlicher Stellung zu führen, dem Schleier des Nichtwissens. Nur so könne ein neutraler Standpunkt und das Einfließen unterschiedlicher Wertvorstellungen garantiert werden. Die Gerechtigkeit liegt folglich in der prozeduralen Gestaltung und Weiterentwicklung des institutionellen Ordnungsrahmens (Brennan \& Buchanan, 2008).

Gerechtigkeit ist mit einem normativen Sollen verbunden. Es betrifft nicht nur die Pflicht gegenüber dem Individuum selbst, sondern auch die Erfüllung der Erwartungen anderer, dass ihnen eine gerechte Behandlung zuteilwird. Handlungen und deren Ergebnisse werden anhand des ethischen Prinzips der Gerechtigkeit bewertet (Göbel, 2013, 144 f.). Auf diese Weise spiegeln sich die geltenden 
sozialen Normen auch in der Gesetzgebung wider und bringen Einstellungen und Werthaltungen einer Gesellschaft zum Ausdruck.

Im Hinblick auf die Themenstellung der Arbeit ist der Gerechtigkeitsaspekt aus der Perspektive der Mütter in Bezug auf die Verteilung von Zeitressourcen sowie Arbeitsleistung und Anerkennung zu untersuchen. Die prozedurale Komponente erscheint besonders aus der kindlichen Perspektive hinsichtlich der Fremdbetreuung wichtig und soll neben der Bedürfnisgerechtigkeit ebenfalls untersucht werden, die auch aus Sicht der Mütter interessant erscheint. Auf diese Weise kann eine Bewertungsgrundlage von Wohlfahrtseffekten geschaffen werden, die einen Rückschluss auf Nachhaltigkeit zulässt. Ausgehend von dem ethisch-moralischen Prinzip der Gerechtigkeit befasst sich der kommende Abschnitt mit der moralischen Urteilsfindung.

\subsubsection{Bildung moralischer Urteile}

Das Treffen moralischer Entscheidungen erfordert die Einnahme eines unparteiischen und objektiven Standpunkts, der gleichermaßen eigene wie fremde Bedürfnisse einbezieht (Fenner, 2008, 10). Die moralische Entscheidung steht in individueller Verantwortung (Göbel, 2013, 49).

Die Moralpsychologie untersucht die Frage, wie Menschen moralische Entscheidungen treffen (Fetchenhauer, 2011, 345). Zunächst begründeten Piaget (1973) sowie Kohlberg (1974) rationalistische, entwicklungspsychologisch orientierte Ansätze und legten ein Phasen- bzw. Stufenmodell der Moralentwicklung vor, dass Kinder entsprechend ihrer Fähigkeiten zur Perspektivübernahme, beginnend mit dem dritten Lebensjahr (Berk, 2011, 453 f.), hierarchisch durchlaufen. Nach diesem sind Kinder in ihrem Urteil zuerst an der Erwartung von Belohnung und Bestrafung orientiert und entsprechen in einer weiteren Phase geltenden gesellschaftlichen Erwartungen. Zuletzt folgt das Urteil dem eigenen Gewissen in Abwägung allgemeingültiger Prinzipien (Noll, 2013, 73 f.). Diese Stufe kann erst mit der Adoleszenz erreicht werden.

Haidt (2001, 2012) begründete den deskriptiven Ansatz des moralischen Intuitionismus. Danach erfolgen moralische Urteile automatisch und intuitiv aufgrund spontaner moralischer Emotionen, die erst im Nachhinein kognitiv begründet werden. Nach seinem Moral Intuitionist Model kann der Prozess der Entscheidungsfindung in sechs Vorgänge unterschieden werden, wobei die ersten vier Prozessschritte der Intuition und die beiden letzten der Rationalität unterliegen, die damit dem Ansatz von Piaget und Kohlberg entsprechen. Nach Haidt (2001, 2012) laufen folgende Schritte ab: (1) Entstehung eines intuitiven Moralurteils 
aufgrund einer automatischen und impliziten Bewertung, (2) die nachträgliche, argumentative Begründung des Urteils, die mit Anstrengung verbunden ist, (3) die verbale Mitteilung und Rechtfertigung der Überzeugung, die hauptsächlich durch die damit ausgelösten Affekte erfolgt und (4) die direkte Beeinflussung durch soziale Gruppennormen. Eher selten findet eine (5) private logische Begründung statt, die die Intuition außer Kraft setzten kann und eine doppelte Einstellung ermöglicht (Wilson, Lindsey \& Schooler, 2000) sowie ein (6) eigenständiger innerer Dialog unter Perspektivübernahme anderer Standpunkte.

Haidt betont zum einen den bedeutenden Einfluss sozialer und kultureller Normen (Haidt, 2001, 819; Haidt, 2012, 56) auf die individuellen Moralurteile. Zum anderen stellt er fest, dass moralische Handlungen in größerem Maße von moralischen Emotionen (Haidt, 2003) determiniert sind als von moralischen Urteilen (Haidt, 2001, 823).

Um die Fragestellung der Arbeit zu untersuchen, ist die moralische Urteilsbildung in vielfacher Hinsicht wichtig. Eine Entscheidung für die Fremdbetreuung der Kinder geht mit einem moralischen Urteil der Mütter einher, das durch soziale Normen beeinflusst wird und für die sie letztendlich die Verantwortung tragen. Moralische Emotionen und die Perspektivübernahmefähigkeit spielen dabei eine wichtige Rolle. Eine Betrachtung der noch fehlenden kindlichen Fähigkeit zur Perspektivübernahme im Hinblick auf die nonverbale Kommunikation und die ebenfalls noch fehlende Kompetenz zur eigenen Emotionsregulation soll helfen, die Fragestellung zu beantworten.

Um einen Überblick zu ermöglichen, soll im folgenden Kapitel der aktuelle Forschungsstand dargelegt werden. Dazu werden zuerst die Ergebnisse einiger Studien vorgestellt und in zwei weiteren Abschnitten durch den Status Quo der Erwerbsarbeit von Müttern und der institutionellen Fremdbetreuung von Kleinkindern ergänzt. 
Open Access Dieses Kapitel wird unter der Creative Commons Namensnennung 4.0 International Lizenz (http://creativecommons.org/licenses/by/4.0/deed.de) veröffentlicht, welche die Nutzung, Vervielfältigung, Bearbeitung, Verbreitung und Wiedergabe in jeglichem Medium und Format erlaubt, sofern Sie den/die ursprünglichen Autor(en) und die Quelle ordnungsgemäß nennen, einen Link zur Creative Commons Lizenz beifügen und angeben, ob Änderungen vorgenommen wurden.

Die in diesem Kapitel enthaltenen Bilder und sonstiges Drittmaterial unterliegen ebenfalls der genannten Creative Commons Lizenz, sofern sich aus der Abbildungslegende nichts anderes ergibt. Sofern das betreffende Material nicht unter der genannten Creative Commons Lizenz steht und die betreffende Handlung nicht nach gesetzlichen Vorschriften erlaubt ist, ist für die oben aufgeführten Weiterverwendungen des Materials die Einwilligung des jeweiligen Rechteinhabers einzuholen.

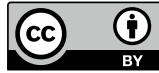

